\title{
Diffusion of silicon in crystalline germanium
}

\section{H. H. Silvestri}

Department of Materials Science and Engineering, University of California, Berkeley, Berkeley, CA 94720 and Materials Sciences Division, Lawrence Berkeley National Laboratory, Berkeley, CA 94720.

\section{H. Bracht}

Institüt für Materialphysik, Universtität Münster, D-48149 Münster, Germany.

\section{J. Lundsgaard Hansen and A. Nylandsted Larsen}

Institute of Physics and Astronomy, University of Aarhus, DK-8000 Aarhus C, Denmark.

\section{E. E. Haller}

Department of Materials Science and Engineering, University of California, Berkeley, Berkeley, CA 94720 and Materials Sciences Division, Lawrence Berkeley National Laboratory, Berkeley, CA 94720.

(Received

\section{ABSTRACT}

We report the determination of the diffusion coefficient of Si in crystalline Ge over the temperature range of 550 to $900{ }^{\circ} \mathrm{C}$. A molecular beam epitaxy (MBE) grown buried Si layer in an epitaxial Ge layer on a crystalline Ge substrate was used as the source for the diffusion experiments. For samples annealed at temperatures above $700{ }^{\circ} \mathrm{C}$, a $50 \mathrm{~nm}$ thick $\mathrm{SiO}_{2}$ cap layer was deposited to prevent decomposition of the Ge surface. We found the temperature dependence of the diffusion coefficient to be described by a single activation energy $(3.32 \mathrm{eV})$ and pre-factor $\left(38 \mathrm{~cm}^{2} / \mathrm{s}\right)$ over the entire temperature range studied. The diffusion of the isovalent Si in Ge is slower than Ge self-diffusion over the full temperature range and reveals an activation enthalpy which is higher than that of selfdiffusion. This points to a reduced interaction potential between the Si atom and the 
native defect mediating the diffusion process. For $\mathrm{Si}$, which is smaller in size than the Ge self-atom, a reduced interaction is expected for a Si-vacancy $\left(\mathrm{Si}-\mathrm{V}_{\mathrm{Ge}}\right)$ pair. Therefore we conclude that Si diffuses in Ge via the vacancy mechanism.

PACS numbers: 66.30.Jt, 61.72.Ji 


\section{INTRODUCTION}

The incorporation of Ge into modern Si based electronics has generated a renewed interest in the detailed understanding of the fundamental properties of Ge. One of the most basic of these properties, and one that is of great importance to the fabrication of electronic devices, is diffusion. The early work of diffusion in Ge focused on dopant diffusion $^{1-3}$ and self-diffusion ${ }^{4-7}$. Werner, et al., ${ }^{8}$ determined a single activation energy of $3.09 \mathrm{eV}$ for self-diffusion in $\mathrm{Ge}$ for the temperature range of $535^{\circ} \mathrm{C}$ to $904{ }^{\circ} \mathrm{C}$. The impact of doping on Ge self-diffusion revealed an enhanced self-diffusion in n-type material and retarded self-diffusion in p-type material. ${ }^{6,9}$ Additionally, Werner, et al., studied the effect of hydrostatic pressure on self-diffusion and deduced an activation volume smaller than one atomic volume for the defect mediating Ge diffusion. The results of doping and hydrostatic pressure on self-diffusion as well as the excellent agreement between the Ge self-diffusion coefficient and the contribution of vacancies to self-diffusion deduced from $\mathrm{Cu}$ diffusion in $\mathrm{Ge},{ }^{10,11}$ provide strong evidence that Ge selfdiffusion is mediated by vacancies in neutral and singly negative charge states.

One element whose diffusion in Ge has received little attention is Si. With the increasing interest to use SiGe epitaxial layers in Si-based integrated circuit technology, the diffusion of $\mathrm{Si}$ in $\mathrm{Ge}$ and $\mathrm{Ge}$ in $\mathrm{Si}$ are of fundamental importance. The diffusion of $\mathrm{Ge}$ in $\mathrm{Si}$ has been studied in greater detail than the diffusion of Si in Ge. ${ }^{12}$ The primary reason for this is prevalence of radiotracer diffusion as the principal experimental diffusion technique for most of the $20^{\text {th }}$ century. As a result of the longer half-life of the

${ }^{71} \mathrm{Ge}$ radioactive isotope (11.2 days) compared to that of ${ }^{31} \mathrm{Si}(2.6 \mathrm{hrs})$, radiotracer 
diffusion experiments with ${ }^{31} \mathrm{Si}$ are more limited than those using ${ }^{71} \mathrm{Ge}$ isotopes. With the recent advances in mass spectrometry-based depth profiling techniques, radiotracers are no longer necessary and stable isotopes may be used as diffusion tracers, removing the limitations of radiotracer half-lives.

Previous studies of the diffusion coefficient of Si in Ge used a wide range of techniques for measuring the diffusion profiles. Räisänen, et al., used ${ }^{30} \mathrm{Si}$ implanted into germanium, along with the ${ }^{30} \mathrm{Si}(\mathrm{p}, \gamma)^{31} \mathrm{P}$ resonance broadening technique. ${ }^{13}$ In the temperature range of 650 to $900{ }^{\circ} \mathrm{C}$, they found an activation enthalpy for Si diffusion in $\mathrm{Ge}$ of $2.9 \mathrm{eV}$ and an exponential pre-factor of $0.24 \mathrm{~cm}^{2} / \mathrm{s}$. More recently, Si diffusion in $\mathrm{Ge}$ in the temperature range of 650 to $950{ }^{\circ} \mathrm{C}$ was measured via secondary ion mass spectrometry (SIMS) from a Si surface layer deposited on a Ge wafer. These authors found an activation enthalpy of $3.47 \pm 0.07 \mathrm{eV}$ and a pre-factor of $140 \pm 50 \mathrm{~cm}^{2} / \mathrm{s}^{14} \mathrm{Using}$ the implantation of the radiotracer ${ }^{31} \mathrm{Si}$ into $\mathrm{Ge}$ and in-situ annealing and ion beam sectioning to determine the depth profiles, Strohm, et al., ${ }^{15}$ found an activation energy of $3.19 \mathrm{eV}$ and a pre-factor of $43 \mathrm{~cm}^{2} / \mathrm{s}$. Si implantation into Ge and SIMS analysis was also performed in the temperature range of 750 to $890{ }^{\circ} \mathrm{C}$ by Uppal, ${ }^{16}$ who found an activation energy of $3.18 \mathrm{eV}$ and pre-factor of $9.7 \mathrm{~cm}^{2} / \mathrm{s}$.

While the above results yield fairly consistent values for the activation energy of Si diffusion in Ge, they were obtained from experiments involving either diffusion from the surface or from an implanted source. Since surfaces and ion implantation are known to affect the equilibrium concentrations of native defects, a measurement approach independent of these possible factors is desirable in order to obtain the true equilibrium diffusion coefficient of $\mathrm{Si}$ in $\mathrm{Ge}$. To avoid these potential sources of non-equilibrium 
diffusion, we determined the diffusion coefficient of Si in Ge from Si diffusion profiles originating from a buried Si layer in an MBE grown Ge epilayer, measured with SIMS. We then compare our results with that of Ge self-diffusion over the same temperature range. This comparison will enable us to draw some conclusions about the defect interactions and mechanisms for Si diffusion in Ge.

\section{EXPERIMENTAL}

In order to form the structures for our diffusion experiments, a $650 \mathrm{~nm}$ thick epitaxial layer of natural germanium was grown on a (001) germanium substrate via $\mathrm{MBE}$ at a growth temperature of $400^{\circ} \mathrm{C}$. During growth, a $150 \mathrm{~nm}$ thick spike of natural Si with a concentration of $\sim 10^{20} \mathrm{~cm}^{-3}$ was incorporated into the epitaxial Ge layer. Due to the decomposition of the Ge surface at high temperatures, all samples annealed at and above $700{ }^{\circ} \mathrm{C}$ were capped with a $50 \mathrm{~nm}$ thick layer of $\mathrm{SiO}_{2}$. The $\mathrm{SiO}_{2}$ cap layer was formed via chemical vapor deposition (CVD) at $450{ }^{\circ} \mathrm{C}$. Atomic force microscopy (AFM) results after $\mathrm{SiO}_{2}$ deposition and after annealing showed no significant increase in surface roughness. In order to reduce the complexity of the SIMS analysis, the $\mathrm{SiO}_{2}$ cap layers were etched off prior to SIMS using a 49\% HF solution for 1 minute. The HF etch did not affect the Ge surface.

Diffusion experiments were performed over the temperature range of $550{ }^{\circ} \mathrm{C}$ to $900{ }^{\circ} \mathrm{C}$. The samples were diced into $5 \times 5 \mathrm{~mm}^{2}$ pieces and cleaned in heated xylene and acetone and room temperature methanol. Residual native oxide was removed from the sample surfaces with a 30 second etch in $\mathrm{HF}\left(\mathrm{SiO}_{2}\right.$ capped samples were not etched prior to annealing). Each individual sample was sealed in a silica ampoule under 0.5 atm Ar, 
to aid thermal transport and to prevent oxidation, and annealed in a resistance-heated tube furnace. A type-S, Pt-PtRh, thermocouple was used to monitor the temperature with an accuracy of $\pm 2{ }^{\circ} \mathrm{C}$. The ampoules were quenched in water to terminate the diffusion. Depth profiles for ${ }^{28} \mathrm{Si}$ were measured via SIMS on a Cameca $4 \mathrm{f}$ at MAS, Inc. using a primary beam of $\mathrm{O}_{2}{ }^{+}$ions with an energy of $3 \mathrm{keV}$.

The diffusion coefficient of Si in Ge was determined by fitting a numerical solution of the single one-dimensional Fick's Law diffusion equation to the experimental Si profile. The as-grown SIMS profile was taken into account as the initial Si distribution. The numerical solution was calculated by means of the partial differential equation solver $\mathrm{ZOMBIE}^{17}$ with a concentration independent diffusion coefficient. Fitting of the simulation to the experimental SIMS data was achieved by a Modified Damped Least Squares non-linear parameter optimization technique (Levenberg-Marquart method) utilizing the mathematical equation solver Profile. ${ }^{18}$ The fitting parameter was the Si diffusion coefficient.

\section{RESULTS AND DISCUSSION}

The measured SIMS depth profiles along with the best fits to the model are presented in Figure 1. Several interesting features show up in the depth profiles. First, in Figure 1(b) a spike of Si is evident at a depth of approximately $650 \mathrm{~nm}$, which coincides with the substrate/epilayer interface. This Si spike is present in all profiles including the as-grown. It should also be noted that no significant out-diffusion from this Si peak is observed, even at the highest temperature $\left(900^{\circ} \mathrm{C}\right)$. Another feature seen in the high temperature profiles of Figure 1(b) is the apparent build-up of Si at the top of the Si 
doped layer, at a depth of approximately $240 \mathrm{~nm}$. This feature is not seen in the as-grown or lower temperature profiles. Subsequent SIMS analysis of the samples for C and O content revealed $\mathrm{C}$ and $\mathrm{O}$ spikes at the same depth as the substrate/epilayer interface, indicating that carbon and oxygen may be responsible for trapping the $\mathrm{Si}$ at the interface. The origin of the $\mathrm{Si}, \mathrm{O}$, and $\mathrm{C}$ at the substrate/epilayer interface is most likely due to contamination at the start of the growth process. No carbon was observed by SIMS at any other point in the epilayer, ruling out carbon contamination as a source of the Si pileup around $240 \mathrm{~nm}$. However, $\mathrm{O}$ contamination is observed in the near-surface region of the as-grown sample and an O pile-up coinciding with the Si pile-up is observed in the samples after diffusion. Two of the O profiles from the diffused samples are shown in Figure 2 to illustrate the effect. The oxygen pile-up is most likely due to an oxygen contamination at the surface. As a result, the oxygen diffuses into the epilayer during annealing and is trapped by the $\mathrm{Si}$, causing the observed pile-up.

Cross-sectional transmission electron microscopy (TEM) was performed on the as-grown sample to determine if structural defects were responsible for the observed features. The TEM images from the as-grown sample are presented in Figure 3. The only observable feature in the TEM image of the epilayer is a series of clusters at the substrate/epilayer interface. Figure 3(b) is a high resolution TEM image of the substrate/epilayer interface showing the clusters. Even though the TEM image does not yield elemental analysis, the TEM image in conjunction with the SIMS data points towards the presence of $\mathrm{SiC}$ precipitates at the substrate/epilayer interface. While it appears that $\mathrm{O}$ is responsible for the Si pile-up around $240 \mathrm{~nm}$, accurate fits to the depth profiles beyond this feature are achieved with the 1-D Fick's Law diffusion 
equation. This is to be expected as evidenced by Figure 2, which shows that the $\mathrm{O}$ is trapped at the front of the Si layer and will not affect the diffusion of Si into the structure. The coefficients for Si diffusion in Ge generated from the fits to the depth profiles are presented in Table I and the temperature dependence is shown in Figure 4. Also shown in Figure 4 are the temperature dependencies of the previous measurements of the diffusivity of $\mathrm{Si}$ in $\mathrm{Ge}^{14-16}$ and Ge self-diffusion. ${ }^{8}$

The temperature dependence of the data presented in this work can be accurately described with a single activation energy $(3.32 \mathrm{eV})$ and pre-factor $\left(38 \mathrm{~cm}^{2} / \mathrm{s}\right)$ over the entire temperature range studied. The values from the current work are given in Table II along with the values from literature. From Figure 4, it is evident that the current work agrees well with the previous measurements of S. Uppal. ${ }^{16}$ However the extension of our results to lower temperatures has expanded the range of the Si diffusivity data by 2 orders of magnitude, which provides increased accuracy for the activation enthalpy of $\mathrm{Si}$ diffusion. The activation energy and pre-factor obtained for Si diffusion in Ge are both greater than those of self-diffusion in Ge. ${ }^{8}$ This yields a diffusivity of $\mathrm{Si}$ in Ge that is slower than that of Ge self-diffusion.

The difference between the self-diffusion activation energy and the impurity diffusion activation energy was used by $\mathrm{Hu}^{19}$ to define the potential between an impuritydefect pair. The smaller activation energy for impurity diffusion yielded an attractive potential between the impurity-defect pair. For $\mathrm{Si}$ in $\mathrm{Ge}$, the larger activation energy and slower Si diffusivity point to a repulsive interaction potential. Both dopant and selfdiffusion in Ge are generally considered to proceed via vacancies. Therefore, if vacancies are the main entity controlling the diffusion in Ge our results suggest a 
repulsive interaction between $\mathrm{Si}$ and the vacancy in Ge. This is consistent with the expected effect of the size difference between the Si atom and the Ge host atoms. The substitutional $\mathrm{Si}$ is smaller than the Ge lattice atom, resulting in a less attractive interaction with vacancies. This reduced attraction is responsible for the slightly higher activation energy for Si diffusion.

\section{CONCLUSIONS}

While there appears to be $\mathrm{O}$ contamination in the near surface region, the SIMS results indicate that $\mathrm{O}$ remains at the top of the $\mathrm{Si}$ layer. This leaves the Si diffusion below that interface free from possible contamination from the oxygen. The depth profiles were accurately described with a single parameter one-dimensional Fick's law diffusion equation and yielded a temperature dependence with a single activation energy over the entire range ( 6 orders of magnitude in diffusivity). This confirms that the $\mathrm{O}$ contamination does not affect significantly the reliability of our data.

The Si diffusion coefficient in Ge is lower than the Ge self-diffusion coefficient

for the entire temperature range. The activation energy and pre-factor of Si diffusion are higher than the corresponding data of Ge self-diffusion. The higher activation energy is consistent with a vacancy-assisted mechanism for Si diffusion in Ge.

\section{ACKNOWLEDGEMENTS}

We would like to thank Z. Liliental-Weber and D. Zakharov of the National Center for Electron Microscopy (NCEM) at the Lawrence Berkeley National Lab for the TEM results. This work was supported in part by a UC Discovery Grant from the 
Industry-University Cooperative Research Program (IUCRP), by US NSF Grant No.

DMR-0405472, by the Director, Office of Science, Office of Basic Energy Sciences,

Division of Materials Sciences and Engineering, of the U.S. Department of Energy under

Contract No. DE-AC03-76SF00098, and by the Danish Natural Science Research

Council. One of us (H. B.) acknowledges support from the Deutsche

Forschungsgemeinschaft. 


\section{REFERENCES}

1. C.S. Fuller, Phys. Rev. 86, 136 (1952).

2. W.C. Dunlap, Jr., Phys. Rev. 94, 1531 (1954).

3. W. Bosenberg, Z. Naturf. 10a, 285 (1955).

4. H. Letaw, L. Slifkin, and W.M. Portnoy, Phys. Rev. 102, 636 (1956).

5. H. Widmer and G.R. Gunther-Mohr, Helv. Phys. Acta 34, 635 (1961).

6. M.M. Valenta and C. Ramasastry, Phys. Rev. 106, 73 (1957).

7. H.D. Fuchs, W. Walukiewicz, E.E. Haller, W. Dondl, R. Schorer, G. Abstreiter, A.I. Rudnev, A.V. Tikhomirov, V.I. Ozhogin, Phys. Rev. B 51, 16817 (1995).

8. M. Werner, H. Mehrer, and H.D. Hochheimer, Phys. Rev. B 32, 3930 (1985).

9. G. Vogel, G. Hettich, and H. Mehrer, J. Phys. C 16, 6197 (1983).

10. N.A. Stolwijk, W. Frank, J. Hölzl, S.J. Pearton, E.E Haller, J. Appl. Phys. 57, 5211 (1985).

11. H. Bracht, N.A. Stolwijk, H. Mehrer, Phys. Rev. B 43, 14465 (1991).

12. N.A. Stolwijk and H. Bracht, "Diffusion in Silicon, Germanium and their Alloys," in Landolt Börnstein New Series Vol. III/33 Subvolume A (Springer), pp. 1-257 (1998).

13. J. Räisänen, J. Hirvonen, and A. Anttila, Solid-State Electronics 24, 333 (1981).

14. U. Södervall and M. Friesel, Defect and Diffusion Forum 143-147, 1053 (1997).

15. A. Strohm, T. Voss, W. Frank, P. Laitinen, J. Räisänen, Z. Metallkd. 93, 737 (2002).

16. S. Uppal, Ph.D. thesis, University of Southampton (2003).

17. W. Jüngling, P. Pichler, S. Selberherr, E. Guerro, and H.W. Pötzl, IEEE Trans. Electron. Devices ED 32, 156 (1985).

18. G.L.J. Ouwerling, doctoral thesis, Delft University of Technology, (1989). 
19. S.M. Hu, Phys. Stat. Sol.(b) 60, 595 (1973). 


\section{$\underline{\text { Table Captions }}$}

Table I. Experimentally determined diffusivity values for $\mathrm{Si}$ in Ge.

Table II. Values for the activation energy and pre-factor for Si diffusion in Ge. 
Table I.

\begin{tabular}{ccc}
\hline \hline Temperature $\left({ }^{\circ} \mathrm{C}\right)$ & Time & Diffusivity $\left(\mathrm{cm}^{2} / \mathrm{s}\right)$ \\
\hline 550 & 30 days & $1.8 \times 10^{-19}$ \\
600 & 5 days & $3.1 \times 10^{-18}$ \\
650 & $24 \mathrm{~h} 21 \mathrm{~min}$ & $2.7 \times 10^{-17}$ \\
700 & $12 \mathrm{~h}$ & $2.1 \times 10^{-16}$ \\
750 & $1 \mathrm{~h}$ & $2.1 \times 10^{-15}$ \\
800 & $30 \mathrm{~min}$ & $1.1 \times 10^{-14}$ \\
850 & $15 \mathrm{~min}$ & $5.0 \times 10^{-14}$ \\
900 & $8 \mathrm{~min}$ & $2.1 \times 10^{-13}$ \\
\hline \hline
\end{tabular}

Table II.

\begin{tabular}{cccc}
\hline \hline Temperature $\left({ }^{\circ} \mathrm{C}\right)$ & $\mathrm{D}_{\mathrm{o}}\left(\mathrm{cm}^{2} / \mathrm{s}\right)$ & $\mathrm{Q}(\mathrm{eV})$ & Reference \\
\hline $650-900$ & 0.24 & 2.9 & {$[13]$} \\
$650-930$ & 140 & 3.47 & {$[14]$} \\
$843-904$ & 43 & 3.19 & {$[15]$} \\
$750-890$ & 9.7 & 3.18 & {$[16]$} \\
$550-900$ & 38 & 3.32 & this work \\
\hline \hline
\end{tabular}




\section{Figure Captions}

Figure 1. SIMS depth profiles of the Si doped Ge epilayer before (dashed line) and after annealing (symbols) along with best fit simulation of the 1-D Fick's Law diffusion equation (solid lines). (a) Samples annealed at $550{ }^{\circ} \mathrm{C}(\mathrm{O}) ; 600{ }^{\circ} \mathrm{C}(\diamond) ; 650{ }^{\circ} \mathrm{C}(\square) ; 700$ ${ }^{\circ} \mathrm{C}(\triangle)$ (b) Samples annealed at $750{ }^{\circ} \mathrm{C}(\mathrm{O}) ; 800{ }^{\circ} \mathrm{C}(\diamond) ; 850{ }^{\circ} \mathrm{C}(\square), 900{ }^{\circ} \mathrm{C}(\triangle)$. For clarity only every third data point is shown in (a) and (b).

Figure 2. SIMS depth profiles of O concentration in the epilayer: as-grown (solid line); after annealing at $550{ }^{\circ} \mathrm{C}(\mathrm{O})$ and $850^{\circ} \mathrm{C}(\square)$. Also shown is the as-grown Si profile (dashed line), for reference.

Figure 3. Cross-sectional Transmission Electron Microscopy (TEM) images of an asgrown Ge epilayer containing a buried Si doped layer. a) a bright field image of the epilayer with sample surface at the top of the image and the growth interface indicated by the dislocation lines (white). The lack of any structural defects beyond the growth interface excludes structural defects as the source of the Si SIMS peak at $240 \mathrm{~nm}$. b) a high resolution TEM image of the growth interface showing the presence of clusters at the growth interface.

Figure 4. Plot of the diffusion coefficient of Si in Ge and Ge self-diffusion ${ }^{8}$ (solid line) as a function of inverse temperature. The closed circles are data points from current work; the thick solid line through the points is a fit to the data. Dashed lines represent 
temperature dependencies reported in literature over the appropriate temperature ranges..$^{14-16}$ 

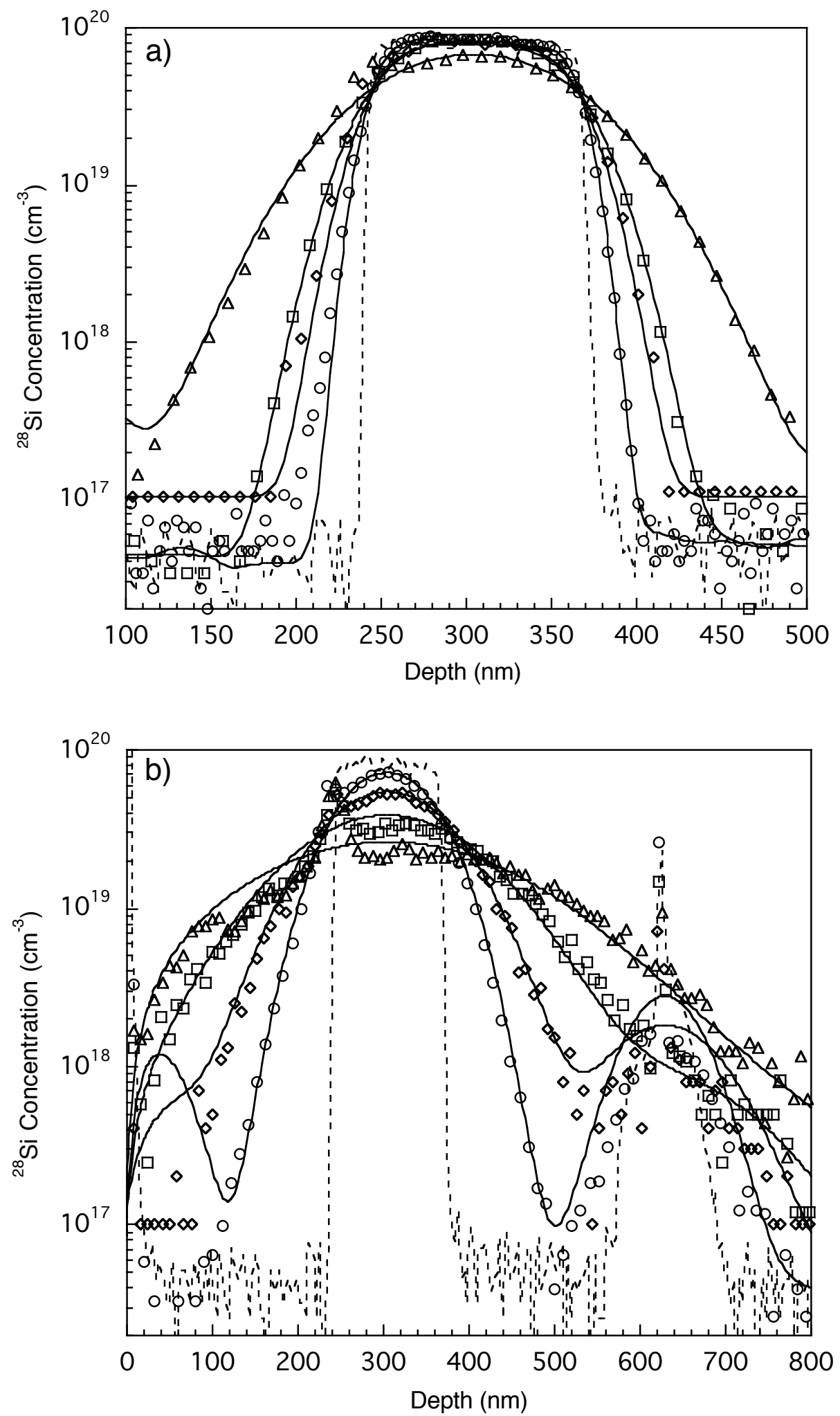

Figure 1 - Silvestri, et al. 


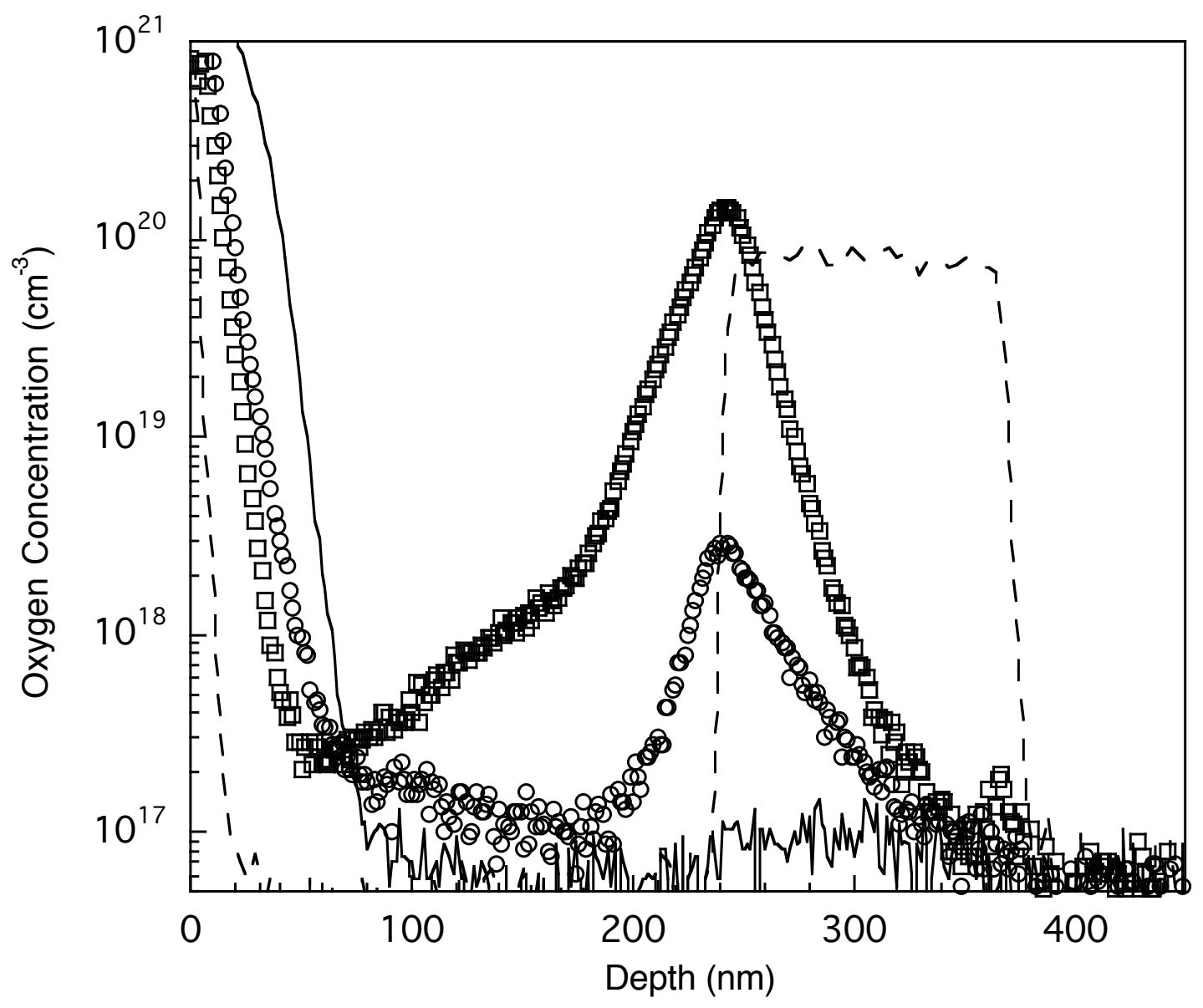

Figure 2 - Silvestri, et al. 

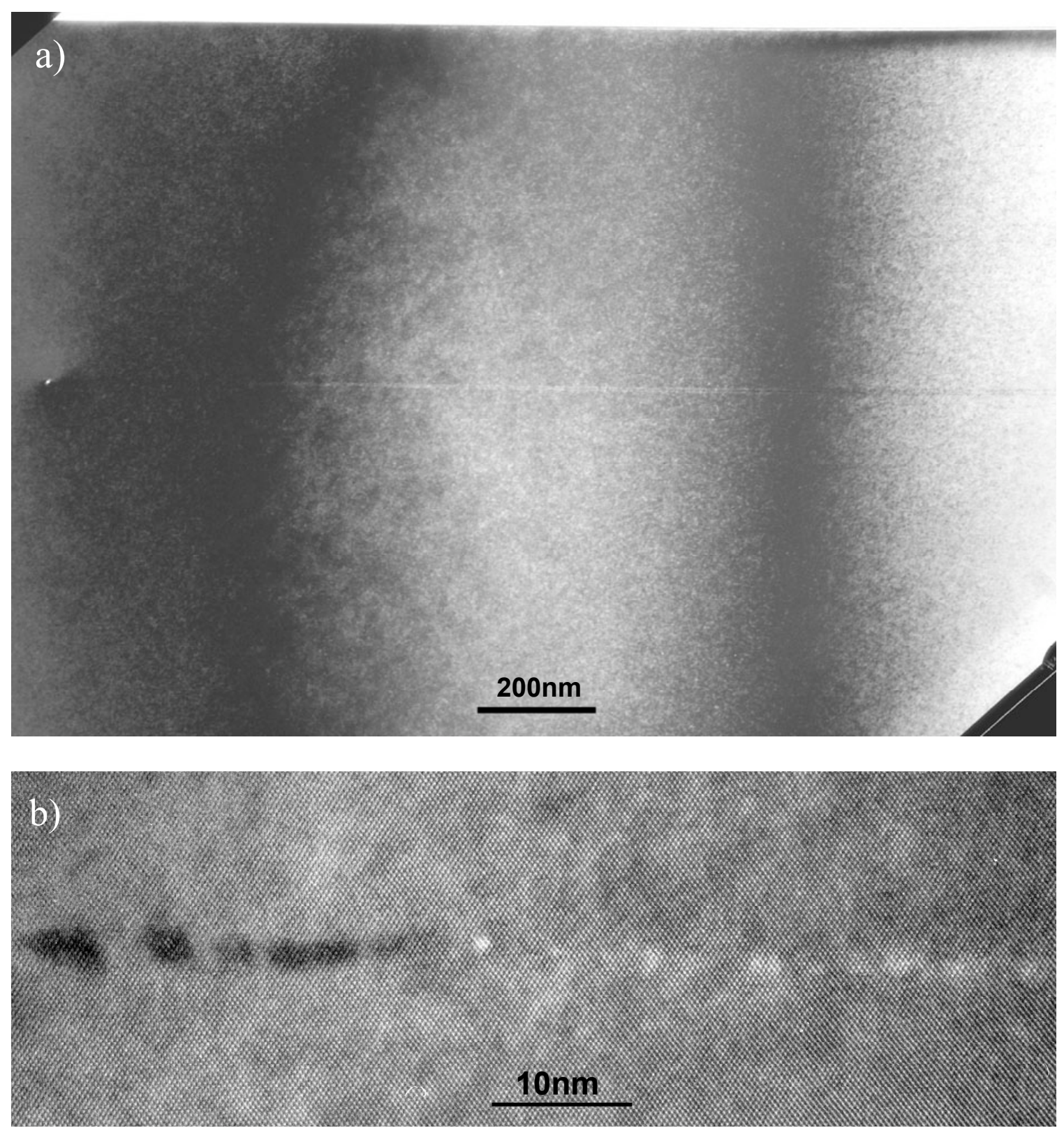

Figure 3 - Silvestri, et al. 


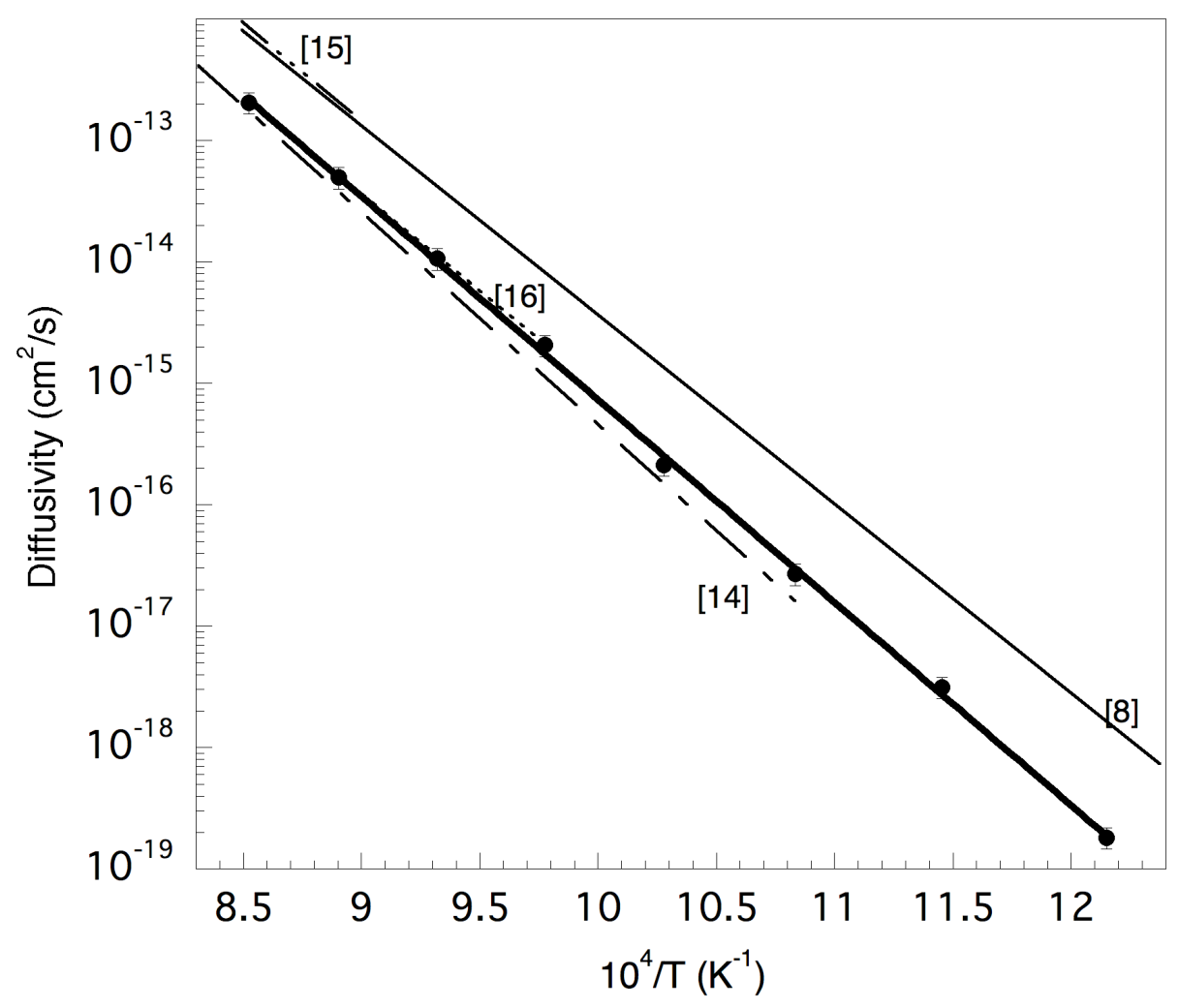

Figure 4 - Silvestri, et al. 